\title{
Effect of Induction Chemotherapy on Swallowing in Head and Neck Cancer Patients
}

\author{
Juliana Portas ${ }^{1}$, Daniela Corrêa ${ }^{2}$, Débora Queija ${ }^{3}$, Lidia Maria Rebolho Batista \\ Arantes $^{4}$, Luciano de Souza Viana ${ }^{4}$, André Lopes Carvalho ${ }^{4 *}$
}

\begin{abstract}
Objective: To evaluate the effect of induction chemotherapy on swallowing and swallowing-related quality of life of patients with oropharyngeal, laryngeal and hypopharyngeal cancer. Study design: Prospective study of 33 patients with locally advanced tumors who were eligible for treatment with neoadjuvant chemotherapy followed by radiotherapy and concurrent chemotherapy. A multidimensional assessment of swallowing was performed using the following tools: (1) Clinical analysis, numerical scale for general pain and painful swallowing, American Speech and Hearing Association (ASHA) Functional Communication Measures (FCM) swallowing assessment scales, (2) assessment of Swallowing-Related Quality of Life (The MD Anderson Dysphagia Inventory - MDADI), and (3) swallowing videofluoroscopy. Results: There was a reduction in general pain scale $(\mathrm{p}=0.021)$, and quality of life (emotional, functional and physical) improved from average limitation (score of 61-80) to minimal limitation (81-100) after induction chemotherapy. According to the swallowing videofluoroscopy results, $26(78.7 \%)$ of the patients had some degree of dysphagia pre-treatment, which decreased to 21 (63.6\%) after induction. Conclusion: Our results suggest that induction chemotherapy improves numerous aspects of swallowing and has a positive impact on the quality of life of pre-chemoradiotherapy patients.
\end{abstract}

Keywords: Swallow- quality of life- induction chemotherapy- head and neck cancer

Asian Pac J Cancer Prev, 20 (1), 91-96

\section{Introduction}

Treatment for advanced head and neck cancer may include radiotherapy, which can be combined with surgery and/or chemotherapy. A guide for clinical practice for the treatment of laryngeal tumors, with the intention of preserving the organ and organ function, was developed by Pfister et al., (2006) based on a multi-institutional study. Chemoradiotherapy is recommended for most T3-T4 patients who only have soft tissue invasion.

The use of neoadjuvant chemotherapy associated with chemoradiotherapy (usually consisting of platinum derivatives, taxanes and fluorouracil) is an option for treating bulky tumors and locally advanced head and neck squamous cell carcinomas (Garden, 2014).

In Pignon et al., (2000) meta-analysis, approximately $75 \%$ of patients exhibited tumor volume reduction in response to neoadjuvant chemotherapy. Radiotherapy is typically combined with sensitizing doses, usually cisplatin, which contributes significantly to therapeutic outcomes. The addition of chemotherapy to combination therapies results in improved functional responses and a $6.5 \%$ increase in overall survival.
Finnegan et al., (2009) report a partial or complete response in $74 \%$ of cases treated with induction chemotherapy, with a local control rate of $89 \%$ after concurrent chemoradiotherapy. The authors report that there is a low incidence of dysphagia, weight loss and need for alternative feeding route at this point of the treatment.

Dysphagia is a common sequela of head and neck cancer and of treatment for these tumors. Caudell et al., (2009) reported that $47 \%$ of patients had some degree of dysphagia prior to the onset of treatment. Painful swallowing, trismus, food aspiration, dependency on an alternative feeding route, weight loss and dietary constraints may become apparent during the diagnosis stage. The anatomic changes caused by the presence of a tumor modify the swallowing physiology. Van der Molen et al., (2009) Patients may present pain, swelling, trismus, mucositis, xerostomia, odynophagia, actinic dermatitis, weight loss and may require the use of an alternative feeding routes after radiotherapy or combined radiotherapy and chemotherapy. Concurrent chemotherapy and radiotherapy can lead to mucositis, nausea, vomiting, stomatitis, fatigue or neutropenia, increasing susceptibility to infection, contributing to an

${ }^{1}$ SLP Head and Neck Department, ${ }^{2}$ Center for Researcher Support, ${ }^{4}$ Head and Neck Oncology Departament, Barretos Cancer Hospital, Barretos-SP, ${ }^{3}$ SLP University of Medicine, São Paulo University, São Paulo-SP, Brazil. *For Correspondence: carvalhoal@gmail.com 
impaired nutritional status and affecting these patients. (Meuric et al., 1999; Colasanto et al., 2005)

A recent study analyzed the effect of neoadjuvant chemotherapy on swallowing physiology, saliva production and quality of life in patients with head and neck tumors. The authors report no significant decrease in swallowing ability or any impact on quality of life after induction chemotherapy (Mittal et al., 2014).

Few studies have investigated the impact of induction chemotherapy alone on swallowing in patients with head and neck cancer. This study was designed based on clinical practice to establish a method for measuring the changes in swallowing noted by the medical staff and reported by patients after induction chemotherapy.

The objective of this study was to evaluate the effect of induction chemotherapy on swallowing and swallowingrelated quality of life of patients with oropharyngeal, laryngeal and hypopharyngeal cancer.

\section{Materials and Methods}

This prospective study was designed to evaluate swallowing and swallowing-related quality of life after induction chemotherapy. Patients from the Barretos Cancer Hospital, Barretos, São Paulo, Brazil with a confirmed histological diagnosis of locally advanced squamous cell carcinoma of the oropharynx, larynx and hypopharynx (T3 or T4), who were eligible for treatment with neoadjuvant chemotherapy followed by radiotherapy and concurrent chemotherapy, were included.

Patients who had previous treatment, cognitive or psychiatric disorders or had been diagnosed with severe laryngeal aspiration were excluded.

The neoadjuvant chemotherapy consisted of intravenous paclitaxel $\left(175 \mathrm{mg} / \mathrm{m}^{2}\right)$ and cisplatin $(80$ $\mathrm{mg} / \mathrm{m} 2$ ) every 21 days for 3 cycles. The treatment was suspended in cases of tumor progression and/or high toxicity.

The study consisted of a swallowing analysis in 33 patients at two time-points: immediately before starting the first induction chemotherapy cycle and on the day of the last cycle of neoadjuvant therapy. The multidimensional swallowing assessment consisted of the following.

\section{Clinical swallowing evaluation}

Swallowing was classified based on the National Outcomes Measurement System (NOMS) Functional Communication Measures (FCM) scale from the American Speech and Hearing Association (ASHA)(2012) This scale assesses speech therapy interventions concerning swallowing. The scale describes various aspects related to swallowing ability that can be evaluated during the course of speech therapy intervention and consists of 7 levels, ranging from the inability to swallow (level 1) to full oral feeding ability. Two other numerical scales were applied: a general pain scale and a painful swallowing scale. The individual stated the level of pain at the moment, from zero (no pain) to 10 (extreme pain). Both scales were dichotomized (using ranges 0-4 and 5-10) for enhanced data analysis.

\section{Evaluation of swallowing-related quality of life}

The MD Anderson Dysphagia Inventory (MDADI), developedby Chen et al., (2001) and validated in Portuguese by Guedes et al., (2013) was used to assess quality of life (QOL). The instrument consists of 20 questions, including one global question and 19 questions subdivided into three domains: emotional, functional and physical. MDADI scores range from 0 to 100 ; the lower the score, the more profound the effect of dysphagia on the patient's quality of life.

\section{Swallowing videofluoroscopy assessment}

To perform the videofluoroscopy assessment, that patients were requested to swallow $5 \mathrm{~mL}$ of barium, 20 $\mathrm{mL}$ of water, $10 \mathrm{~mL}$ of pudding (barium and thickener) and two solids (barium with cookie).

The correlated variables were as follows: (1) stasis in the oral/oropharyngeal cavity; (2) stasis in the hypopharynx; (3) functional swallowing of liquids, pudding and solids; (4) oral, pharyngeal and oropharyngeal dysphagia; (5) the penetration/aspiration scale; and (6) the dysphagia severity scale.

Pudding was the only sample consistency used for the stasis analysis, as it was an intermediate consistency and produced more reliable results. The results were grouped by location, namely oral cavity + oropharynx (stasis on the floor of the mouth, hard palate, tongue, tongue base and vallecula) and hypopharynx (posterior wall of the pharynx, pharyngeal-esophageal transition, arytenoids and piriform cortex) to facilitate the characterization of stasis.

The functional swallowing variables for liquids, pudding and solids were analyzed to determine when they did not exhibit a functional impact on the outcome of the test. Even when changes such as discrete stasis, reduced laryngeal elevation, change in the bolus formation or beginning of the pharyngeal swallowing in the pharyngoesophageal transition and/or spontaneous compensatory maneuvers performed by the patients occurred, swallowing was scored within the normal range.

Laryngeal penetration and aspiration were classified according to the scale described by Rosenbek et al., (1996) The degree of dysphagia was determined based on the severity scale proposed by O'Neil et al., (1999). The tests were randomized and then analyzed by three speech therapists with more than three years of experience in swallowing videofluoroscopy assessment.

This study was approved by the Ethics and Research Committee of the Institution.

\section{Statistical analysis}

The data analysis was performed using the intention-to-treat method. The qualitative variables are expressed as frequencies and percentages, and the continuous variables are expressed as means, standard deviations, medians, and minimum and maximum values. The association between qualitative variables was tested using the McNemar test or the marginal homogeneity test. The continuous variables with normal distribution were compared using paired Student's t-test, and the variables without normal distribution were analyzed using the nonparametric Wilcoxon test. Normality was assessed 
using the Shapiro-Wilks test. SPSS 21.0 software was used to perform the calculations. All tests were evaluated using a significance level of $5 \%$.

\section{Results}

The assessments were performed before and after induction chemotherapy. All 33 patients in the study were from Barretos Cancer Hospital, Barreto, São Paulo, Brazil. Most of the patients were male (90.9\%), aged 37-82 years, and had little education.

The most common primary tumor site was oropharynx (22: $66.7 \%)$, followed by larynx (7: $21.2 \%)$ and hypopharynx (4: 12.1\%). Regarding clinical staging, most of the patients $(22: 66.7 \%)$ were classified as T3, a significant proportion $(90.9 \%)$ had lymph node metastasis, and $28(84.8 \%)$ were classified as clinical stage IV. The clinical and demographic data are detailed in Table 1.

Two of the patients $(6 \%)$ had only one induction cycle, due to the development of renal toxicity in one patient and neutropenia in the other. Four of the patients (12\%) completed two cycles, and 27 patients $(82 \%)$ completed the recommended three cycles.

The effect of neoadjuvant chemotherapy on swallowing and swallowing-related quality of life was assessed via the following variables: pain, painful swallowing, dysphagia clinical scale (assessed by FCM-ASHA), and swallowing-related quality of life (assessed by the MDADI questionnaire: emotional, functional, physical, global and final score domains). The degree of dysphagia, as determined by videofluoroscopy, was defined using the

Table 1. Clinical and Demographics Characteristics of the Patients

\begin{tabular}{lc}
\hline Variable & $\mathrm{N}(\%)$ \\
\hline Gender & $3(9.1)$ \\
$\quad$ Female & $30(90.9)$ \\
Male & \\
Age & $16(48.5)$ \\
$<60$ & $17(51.5)$ \\
$>60$ & \\
Educational level & $20(60.6)$ \\
$\quad<$ incomplete primary education & $13(39.4)$ \\
$>$ complete primary education & \\
Tumor site & $11(33.3)$ \\
Hypopharynx + Larynx & $22(66.7)$ \\
Oropharynx & \\
T Staging & $22(66.7)$ \\
T3 & $11(33.3)$ \\
T4 & \\
N Staging & $3(9.1)$ \\
N0 & $30(90.9)$ \\
N+ & \\
Clinical Staging & $5(15.2)$ \\
III & $28(84.8)$ \\
\hline
\end{tabular}

Table 2. Comparison of the General Pain and Painful Swallowing Scales between the Assessment TimePoints, and $p$-values

\begin{tabular}{lccc}
\hline Variable & $\begin{array}{c}\text { Pre-Induction } \\
\mathrm{N}(\%)\end{array}$ & $\begin{array}{c}\text { Post-Induction } \\
\mathrm{N}(\%)\end{array}$ & $\mathrm{p}$ \\
\hline General Pain Scale & & & \\
$0-4$ & $21(63.6)$ & $29(87.9)$ & 0.021 \\
$5-10$ & $12(36.4)$ & $4(12.1)$ & \\
Painful Swallowing Scale & & \\
$0-4$ & $20(60.6)$ & $27(81.8)$ & 0.065 \\
$5-10$ & $13(39.4)$ & $6(18.2)$ & \\
\hline \multicolumn{4}{l}{ Chi-square or Fisher's exact tests (number of events (\%)) }
\end{tabular}

following variables: stasis in the oral/oropharynx cavity when swallowing pudding, stasis in the hypopharynx when swallowing pudding, laryngeal penetration, laryngeal aspiration and functional swallowing for liquids, pudding and solids.

Table 2 shows the pre- and post-induction results of the general pain and painful swallowing scales. We observed a statistically significant decrease in general pain after induction chemotherapy $(\mathrm{p}=0.021)$.

After treatment, we observed a statistically significant improvement in the swallowing-related quality of life for the emotional, functional and physical domains. The emotional and functional domains improved from average limitation (scores of 61-80) to minimal limitation (81-100). There were no statistically significant differences for the global domain or in the final score (Table 3).

The ASHA clinical evaluation scale indicated improvement in swallowing after chemotherapy. Dysphagia was classified as level 7 (ability to swallow all consistencies) in $13(39.4 \%)$ cases pre-induction and in $17(51.5 \%)$ cases post-induction. However, this difference was not statistically significant, as shown in Table 4.

The dysphagia characterization details are shown in Table 5. Based on the results from the swallowing videofluoroscopy assessment, most of the cases presented some degree of dysphagia pre-treatment (26 patients: $78.7 \%$ ), with 18 cases $(54.5 \%)$ being classified as oropharyngeal. After induction, a total of 21 patients (63.6\%) exhibited dysphagia, with 15 cases $(45.5 \%)$ being classified as oropharyngeal. Treatment resulted in a decrease in the frequency of stasis in the oral/ oropharyngeal cavity and in the occurrence of dysphagia. After the neoadjuvant treatment, more patients were able to swallow solid and liquid consistencies with no

Table 3. Comparison of the Swallowing-Related Quality of Life Domains between the Assessment Time-Points, and $p$-values

\begin{tabular}{lccc}
\hline & Pre-Induction & Post-Induction & $P$ \\
\hline Domain & Mean (SD) & Mean (SD) & \\
Emotional & $70.9( \pm 13.5)$ & $82.1( \pm 13.8)$ & 0.002 \\
Functional & $74.6( \pm 10.6)$ & $83.2( \pm 12.5)$ & 0.005 \\
Physical & $64.7( \pm 14.8)$ & $73.9( \pm 17.3)$ & 0.006 \\
Global & $72.2( \pm 19.9)$ & $75.7( \pm 25.3)$ & 0.643 \\
Final Score & $70.6( \pm 2.8)$ & $78.7( \pm 15.2)$ & 0.19 \\
\hline
\end{tabular}

Asian Pacific Journal of Cancer Prevention, Vol 20 
Table 4. Comparison of ASHA-FCM Scale Levels between Assessment Time-Points, and $p$-values

\begin{tabular}{lccc}
\hline Variable & $\begin{array}{c}\text { Pre-Induction } \\
\mathrm{N}(\%)\end{array}$ & $\begin{array}{c}\text { Post-Induction } \\
\mathrm{N}(\%)\end{array}$ & $\mathrm{p}$ \\
\hline ASHA NOMS & & & 0.157 \\
Level 1/2/3 & $3(9.1)$ & $1(3.0)$ & \\
Level 4/ 5/6 & $17(51.5)$ & $15(45.5)$ & \\
Level 7 & $13(39.4)$ & $17(51.5)$ & \\
\hline
\end{tabular}

Chi-square or Fisher's exact tests (number of events (\%))

Table 5. Comparison of Swallowing Videofluoroscopy Variables between the Assessment Time-Points, and p-values

\begin{tabular}{|c|c|c|c|}
\hline Variable & $\begin{array}{c}\text { Pre-Induction } \\
\mathrm{N}(\%)\end{array}$ & $\begin{array}{c}\text { Post-Induction } \\
\text { N (\%) }\end{array}$ & $\mathrm{p}$ \\
\hline \multicolumn{4}{|l|}{ Dysphagia } \\
\hline None & $7(21.2)$ & $12(36.4)$ & 0.191 \\
\hline Oral & $3(9.1)$ & $1(3.0)$ & \\
\hline Pharyngeal & $5(15.2)$ & $5(15.2)$ & \\
\hline Oropharyngeal & $18(54.5)$ & $15(45.5)$ & \\
\hline \multicolumn{4}{|c|}{ Oral + Oropharyngeal Stasis with Pastes } \\
\hline Yes & $27(81.8)$ & $24(72.7)$ & 0.508 \\
\hline No & $6(18.2)$ & $9(27.3)$ & \\
\hline \multicolumn{4}{|c|}{ Hypopharynx Stasis with Pastes } \\
\hline Yes & $10(76.7)$ & $10(76.7)$ & 0.549 \\
\hline No & $23(23.3)$ & $23(23.3)$ & \\
\hline \multicolumn{4}{|c|}{ Functional Swallowing of Liquids } \\
\hline Yes & $24(72.7)$ & $28(84.8)$ & 0.125 \\
\hline No & $9(27.3)$ & $5(15.2)$ & \\
\hline \multicolumn{4}{|c|}{ Functional Swallowing of Pastes } \\
\hline Yes & $22(66.7)$ & $22(66.7)$ & 1 \\
\hline No & $11(33.3)$ & $11(33.3)$ & \\
\hline \multicolumn{4}{|c|}{ Functional Swallowing of Solids } \\
\hline Yes & $22(66.7)$ & $25(75.8)$ & 0.549 \\
\hline No & $11(33.3)$ & $8(24.2)$ & \\
\hline
\end{tabular}

Chi-square or Fisher's exact tests (number of events (\%))

functional impact.

The dysphagia severity and the results from the penetration and aspiration scales are shown in Table 6 . There were no changes in dysphagia after treatment. Laryngeal penetration occurred in nine pre-chemotherapy cases, and four had aspiration, three of which were silent. There was reduced laryngeal penetration after induction, and the two cases that still exhibited laryngeal aspiration were no longer silent.

\section{Discussion}

Several studies have assessed organ preservation in head and neck tumors in patients who receive induction chemotherapy followed by concurrent chemoradiotherapy. Neoadjuvant treatment regimens that include cisplatin, taxol and fluorouracil have shown high overall response rates. In bulky tumors and/or tumors with local metastasis,
Table 6. Comparison of the Dysphagia Severity Scores, (O'Neil et al., 1999) between the Assessment Time-Points, and $p$-values

\begin{tabular}{|c|c|c|c|}
\hline Scale & $\begin{array}{l}\text { Pre-Induction } \\
\text { N }(\%)\end{array}$ & $\begin{array}{c}\text { Post-Induction } \\
\text { N (\%) }\end{array}$ & $\mathrm{p}$ \\
\hline \multicolumn{4}{|c|}{ Dysphagia Severity (O’Neil) } \\
\hline Normal & $13(39.4)$ & $14(42.5)$ & \\
\hline Mild & $17(51.5)$ & $17(51.5)$ & 0.655 \\
\hline Moderate/Severe & $3(9.1)$ & $2(6)$ & \\
\hline \multicolumn{4}{|c|}{ Penetration and Aspiration (Rosenbek) } \\
\hline Without Penetration & $20(60.6)$ & $24(72.7)$ & \\
\hline Penetration & $9(27.3)$ & $7(21.3)$ & 0.157 \\
\hline Aspiration & $4(12.1)$ & $2(6)$ & \\
\hline
\end{tabular}

there is a decrease in tumor size, reducing the field of radiotherapy and therefore toxicity.

The presence of a tumor can change the anatomy and physiology of swallowing, cause desensitization of the larynx, and obstruct the pharynx, restricting bolus passage. Swallowing ability, efficiency and safety after chemoradiotherapy depend on a number of factors that correlate with the prognosis for dysphagia rehabilitation. These factors include tumor size, changes in swallowing before the treatment, early speech therapy intervention and adherence to treatment ( Salama et al., 2008; Starmer et al., 2014)

Few studies have examined swallowing before cancer treatment. However, it is necessary to understand the starting point and the baseline conditions to fully understand the rehabilitation process throughout treatment.

Likewise, few studies have assessed the effects of chemotherapy alone in swallowing. Mittal et al., (2014) conducted a pilot study that analyzed temporal measures of changes in swallowing physiology and saliva production after induction chemotherapy. The authors emphasized that this treatment did not have a negative impact on swallowing and saliva production.

In the present study, we assessed patients who received induction chemotherapy followed by chemoradiotherapy for advanced tumors (laryngeal, oropharyngeal and hypopharyngeal tumors, stage T3 and T4) with no bone or cartilage invasion. Unlike the study by Mittal et al., (2014) we observed an improvement in some aspects of swallowing after treatment in our clinical practice, such as a reduction in odynophagia. Mittal et al., (2014) used a TPF regimen (taxol, platinum, fluorouracil) for induction, which may explain the difference in the findings, as these drugs are slightly more toxic compared to the combination of cisplatin and paclitaxel.

The study population consisted of patients of both genders, with a wide age range. The vast majority had not finished primary school. The sample included patients with advanced stage laryngeal, hypopharyngeal and oropharyngeal cancer. Mittal et al., (2014) also included patients with unknown primary tumors in both early and advanced stages, and these variables may have negatively influenced the study results. 
We observed a statistically significant reduction in referred pain, both for general pain and painful swallowing, after induction. Nerve decompression or the restored passage of food in the pharynx may occur with a reduction in tumor volume, leading to less discomfort during swallowing and reducing referred pain.

Patients with dysphagia exhibit several signs and symptoms to varying degrees, including coughing or phlegm production during some meals, constraints regarding the consistency of food that can be consumed, lack of appetite and even isolation from society, due to the significant functional disability.

Impaired swallowing effectiveness and safety can have serious consequences for patients' quality of life. (Gustafsson and Tibbling, 2018; Carrara-de Angelis and Bandeira, 2009) report that the restrictions experienced by patients with dysphagia can create feelings of frustration, depression, shame and embarrassment, causing them to no longer eat in public. Thus, the social function of participating in meals/feeding is impaired.

Studying swallowing-related quality of life helps to understand the real impact that the changes arising from dysphagia can have on the physical, mental and social wellbeing of the patient. McHorney et al., (2002)

The evaluation of quality of life specifically related to swallowing facilitates the interpretation of the patient's perception of dysphagia before, during and after treatment. It is essential to know the real impact of the changes experienced while eating to direct management according to the patients' needs and the health professionals' commitment to improved rehabilitation of dysphagia. (Rogers et al., 2002; chen et al., 2009). According to Chen et al., (2009), 23 the limitations in swallowing identified by the MDADI are as follows: 0-20: profound limitation; 21-40: severe limitation; 41-60: moderate limitation; 61-80: average limitation; and 81-100: minimal limitation. This study demonstrates the positive effect of induction chemotherapy for reducing signs and symptoms and for improving swallowing-related quality of life from average to minimal limitation after chemotherapy.

The patients in our study exhibited an improvement in oral ingestion, as evaluated by the ASHA scale, after induction chemotherapy, as some of the patients initially required compensatory strategies, even if spontaneous, during swallowing and were unable to swallow solid foods. The ASHA scale evaluates the effectiveness of oral ingestion and the need for the use of compensatory strategies to enable safe swallowing. Therefore, the ASHA scale is an important tool that detects changes in ability levels after an intervention.

The objective evaluation measures for swallowing were not significantly different in the two assessment time-points. However, there was an improvement after induction for the following variables: dysphagia, stasis in the oral/oropharyngeal cavity and functional swallowing of liquids and solids. These results may reflect the significant reduction in tumor volume, which facilitates swallowing biomechanics, bolus progression and pharynx and larynx sensitivity. Studies by Mittal et al., (2014) and Salamaet al., (2008) report a reduction in food residues as a result of the reduction in tumor volume.
The use of dysphagia severity and penetration and aspiration scales is necessary to compare results based on the same method of analysis; however, despite using swallowing tests and scales as an objective measure of the degree of dysphagia, it is still a subjective measure.

The speech-language literature describe changes in swallowing ability after chemoradiotherapy. However, this study has demonstrated that pre-treatment, some patients aspirate, which is mostly silent, regardless of the existence of swallowing complaints. This indicates the importance of assessment and speech-therapy follow-up as well the importance of performing objective swallowing tests to better identify feeding risks and safety from the start of treatment.

Further studies assessing the effects of induction chemotherapy on swallowing are ongoing. It is important to know all of the benefits that this treatment regimen offers. However, further studies that analyze objective assessments of swallowing and quality of life during this phase of cancer treatment in a larger sample size are still needed.

In conclusion, our results suggest that induction chemotherapy may be beneficial to patients before the onset of radiotherapy, reducing swallowing changes and limitations in quality of life during this phase of cancer treatment.

\section{References}

ASHA (2012). American speech and Hearing Association. National outcomes measurement system (NOMS) [internet]. Unknown site. American speech and Hearing Association; c1997-2012. http://www.asha. org/members/research/noms/. Accessed: 2012 June 12. [ Links ].

Carrara-de Angelis E, Bandeira AK (2009). Qualidade de vida em deglutição. In: Jotz GP, Carrara-de Angelis E, Barros APB, eds. Tratado de deglutição e disfagia no adulto e na criança [Treaty of swallowing and dysphagia in adults and in children]. Rio de Janeiro Revinter, 2009, 364-8.

Caudell JJ, Schaner PE, Meredith RF, et al (2009). Factors associated with long-term dysphagia after definitive radiotherapy for locally advanced head-and-neck cancer. Int J Radiat Oncol Biol Phys, 73, 410-15.

Chen AY, Frankowski R, Bishop-Leone J, et al (2001). The development and validation of a dysphagia-specific quality-of-life questionnaire for patients with head and neck cancer: the M. D. Anderson dysphagia inventory. Arch Otolaryngol Head Neck Surg, 127, 870-6.

Colasanto JM, Prasad P, Nash MA, Decker RH, Wilson LD (2005). Nutritional support of patients undergoing radiation therapy for head and neck cancer. Oncology (Williston Park, N.Y.), 19, 371-9.

Duarte VM, Chhetri DK, Liu YF, Erman AA, Wang MB (2013). Swallow preservation exercises during chemoradiation therapy maintains swallow function. Otolaryngol Head Neck Surg, 149, 878-84.

Finnegan V, Parsons JT, Greene BD, Sharma V (2009). Neoadjuvant chemotherapy followed by concurrent hyperfractionated radiation therapy and sensitizing chemotherapy for locally advanced (T3-T4) oropharyngeal squamous cell carcinoma. Head Neck, 31,167-74.

Garden AS (2014). The never-ending story: finding a role for neoadjuvant chemotherapy in the management of head and neck cancer. $J$ Clin Oncol, 32, 2685-6.

Asian Pacific Journal of Cancer Prevention, Vol 20 
Gillespie MB, Brodsky MB, Day TA, Lee FS, Martin-Harris B (2004). Swallowing-related quality of life after head and neck cancer treatment. Laryngoscope, 114, 1362-7.

Guedes RL, Angelis EC, Chen AY, Kowalski LP, Vartanian JG (2013). Validation and application of the M.D. Anderson Dysphagia Inventory in patients treated for head and neck cancer in Brazil. Dysphagia, 28, 24-32.

Gustafsson B, Tibbling L (1991). Dysphagia, an unrecognized handicap. Dysphagia, 6, 193-9.

McHorney CA, Robbins J, Lomáx K, et al (2002). The SWAL-QOL and SWAL-CARE outcomes tool for oropharyngeal dysphagia in adults: III. Documentation of reliability and validity. Dysphagia, 17, 97-114.

Meuric J, Garabige V, Blanc-Vincent MP, Lallemand Y, Bachmann P (1999). Bonnes pratiques pour la prise en charge diététique des patients atteints de cancer des voies aérodigestives supérieures [Good practices for the dietary management of patients with cancer of the upper aerodigestive tract]. Bull Cancer, 86, 843-54.

Mittal BB, Pauloski BR, Rademaker AW, et al (2014). Effect of induction chemotherapy on swallow physiology and saliva production in patients with head and neck cancer: A pilot study. Head Neck, doi: 10.1002/hed.23635. [Epub ahead of print]

O’Neil KH, Purdy M, Falk J, Gallo L (1999). The dysphagia outcome and severity scale. Dysphagia, 14, 139-45.

Pfister DG, Su YB, Kraus DH, et al (2006). Concurrent cetuximab, cisplatin, and concomitant boost radiotherapy for locoregionally advanced, squamous cell head and neck cancer: a pilot phase II study of a new combined-modality paradigm. J Clin Oncol, 24, 1072-8.

Pignon JP, Bourhis J, Domenge C, Designé L (2000). Chemotherapy added to locoregional treatment for head and neck squamous-cell carcinoma: three meta-analyses of updated individual data. MACH-NC Collaborative Group. Meta-Analysis of Chemotherapy on Head and Neck Cancer. Lancet, 355, 949-55.

Rogers SN, Laher SH, Overend L, Lowe D (2002). Importancerating using the University of Washington quality of life questionnaire in patients treated by primary surgery for oral and oro-pharyngeal cancer. J Craniomaxillofac Surg, 30, 125-32.

Rosenbek JC, Robbins JA, Roecker EB, Coyle JL, Wood JL (1996). A penetration-aspiration scale. Dysphagia, 11, 93-8.

Salama JK, Stenson KM, List MA, et al (2008). Characteristics associated with swallowing changes after concurrent chemotherapy and radiotherapy in patients with head and neck cancer. Arch Otolaryngol Head Neck Surg, 134, 1060-5.

Starmer HM, Yang W, Raval R, et al (2014). Effect of Gabapentin on swallowing during and after Chemoradiation for oropharyngeal squamous cell cancer. Dysphagia, 29, 396-402.

Van der Molen L, van Rossum MA, Ackerstaff AH, et al (2009). Pretreatment organ function in patients with advanced head and neck cancer: clinical outcome measures and patients. BMC Ear Nose Throat Disord, 9, 10.

This work is licensed under a Creative Commons AttributionNon Commercial 4.0 International License. 